\title{
A Banach space with a symmetric basis which is of weak cotype 2 but not of cotype 2
}

\author{
by
}

Peter G. Casazza (Columbia, MO) and Niels J. Nielsen (Odense)

\begin{abstract}
We prove that the symmetric convexified Tsirelson space is of weak cotype 2 but not of cotype 2 .
\end{abstract}

Introduction. Weak type 2 and weak cotype 2 spaces were originally introduced and investigated by V. D. Milman and G. Pisier in [10] and weak Hilbert spaces by Pisier in [13]. A further detailed investigation can be found in Pisier's book [14]. The first example of a weak Hilbert space which is not isomorphic to a Hilbert space is the 2-convexified Tsirelson space (called the convexified Tsirelson space in this paper). This follows from the results of W. B. Johnson in [4]. For a detailed study of the original Tsirelson space we refer to [3].

Let $X$ be a Banach space with a symmetric basis. It was proved in [14] that if $X$ is a weak Hilbert space, then it is isomorphic to a Hilbert space, and this has led to the belief that if $X$ is just of weak cotype 2, then it is of cotype 2 . However, this turns out not necessarily to be the case. The main result of this paper states that the symmetric convexified Tsirelson space is of weak cotype 2 but not of cotype 2 .

We now wish to discuss the arrangement of this paper in greater detail.

In Section 1 we give some basic facts on properties related to weak type 2 and weak cotype 2 while Section 2 is devoted to a review of some results on the convexified Tsirelson space which we need for our main result. Most of these results are stated without proofs since they can be proved in a similar manner to the corresponding results for the original Tsirelson space.

In Section 3 we make the construction of the symmetric convexified Tsirelson space, investigate its basic properties and prove our main result stated above.

2000 Mathematics Subject Classification: 46B03, 46B07.

Supported by NSF grants DMS 9706108 and 0102686.

Supported by the Danish Natural Science Research Council, grant 9801867. 
Acknowledgements. The authors are indebted to Nigel Kalton for communicating Theorem 3.4 to us. Also, the authors are indebted to the referee for a very careful reading of the manuscript and many important suggestions for improvement.

1. Notation and preliminaries. In this paper we will use the notation and terminology commonly used in Banach space theory as it appears in [8], [9] and [16]. $B_{X}$ will always denote the closed unit ball of the Banach space $X$, and if $X$ and $Y$ are Banach spaces, then $B(X, Y)$ $(B(X)=B(X, X))$ denotes the space of all bounded linear operators from $X$ to $Y$.

We let $\left(g_{n}\right)$ denote a sequence of independent standard Gaussian variables on a fixed probability space $(\Omega, \mathcal{S}, \mu)$ and recall that a Banach space $X$ is said to be of type $p, 1 \leq p \leq 2$, respectively cotype $p, 2 \leq p<\infty$, if there is a constant $K \geq 1$ so that for all finite sets $\left\{x_{1}, \ldots, x_{n}\right\} \subseteq X$ we have

respectively

$$
\left(\int\left\|\sum_{j=1}^{n} g_{j}(t) x_{j}\right\|^{p} d \mu(t)\right)^{1 / p} \leq K\left(\sum_{j=1}^{n}\left\|x_{j}\right\|^{p}\right)^{1 / p},
$$

$$
\left(\sum_{j=1}^{n}\left\|x_{j}\right\|^{p}\right)^{1 / p} \leq K\left(\int\left\|\sum_{j=1}^{n} g_{j}(t) x_{j}\right\|^{p} d \mu(t)\right)^{1 / p} .
$$

The smallest constant $K$ which can be used in (1.1) (respectively (1.2)) is denoted by $K^{p}(X)$ (respectively $\left.K_{p}(X)\right)$.

If $L$ is a Banach lattice and $1 \leq p<\infty$, then $L$ is said to be $p$-convex, respectively $p$-concave, if there is a constant $C \geq 1$ so that for all finite sets $\left\{x_{1}, \ldots, x_{n}\right\} \subseteq L$ we have

respectively

$$
\left\|\left(\sum_{j=1}^{n}\left|x_{j}\right|^{p}\right)^{1 / p}\right\| \leq C\left(\sum_{j=1}^{n}\left\|x_{j}\right\|^{p}\right)^{1 / p}
$$

$$
\left(\sum_{j=1}^{n}\left\|x_{j}\right\|^{p}\right)^{1 / p} \leq C\left\|\left(\sum_{j=1}^{n}\left|x_{j}\right|^{p}\right)^{1 / p}\right\| .
$$

The smallest constant $C$ which can be used in (1.3) (respectively (1.4)) is denoted by $C^{p}(L)$ (respectively $\left.C_{p}(L)\right)$.

It follows from $[9,1 . d .6(i)]$ that if $L$ is of finite concavity (equivalently of finite cotype), then there is a constant $K \geq 1$ so that

$$
\begin{aligned}
\frac{1}{K}\left\|\left(\sum_{j=1}^{n}\left|x_{j}\right|^{2}\right)^{1 / 2}\right\| & \leq\left(\int\left\|\sum_{j=1}^{n} g_{j}(t) x_{j}\right\|^{2} d \mu(t)\right)^{1 / 2} \\
& \leq K\left\|\left(\sum_{j=1}^{n}\left|x_{j}\right|^{2}\right)^{1 / 2}\right\| .
\end{aligned}
$$


A Banach space $X$ is said to be of weak type 2 if there is a constant $C$ and a $\delta, 0<\delta<1$, so that whenever $E \subseteq X$ is a subspace, $n \in \mathbb{N}$ and $T \in B\left(E, \ell_{2}^{n}\right)$, then there is an orthogonal projection $P$ on $\ell_{2}^{n}$ of rank larger than $\delta n$ and an operator $S \in B\left(X, \ell_{2}^{n}\right)$ with $S x=P T x$ for all $x \in E$ and $\|S\| \leq C\|T\|$.

Similarly $X$ is of weak cotype 2 if there is a constant $C$ and a $\delta, 0<\delta<1$, so that whenever $E \subseteq X$ is a finite-dimensional subspace, then there is a subspace $F \subseteq E$ so that $\operatorname{dim} F \geq \delta \operatorname{dim} E$ and $d\left(F, \ell_{2}^{\operatorname{dim} F}\right) \leq C$.

Our definitions of weak type 2 and weak cotype 2 spaces are not the original ones, but are chosen out of the many equivalent characterizations given by Pisier [14].

A weak Hilbert space is a space which is both of weak type 2 and weak cotype 2 .

If $A$ is a set we let $|A|$ denote the cardinality of $A$.

Definition 1.1. If $\left(x_{n}\right)$ and $\left(y_{n}\right)$ are sequences in a Banach space $X$, we say that $\left(x_{n}\right)$ is $K$-dominated by $\left(y_{n}\right)$ if there is a constant $K>0$ so that for all finitely non-zero sequences $\left(a_{n}\right)$ of scalars we have

$$
\left\|\sum_{n} a_{n} x_{n}\right\| \leq K\left\|\sum_{n} a_{n} y_{n}\right\| .
$$

The sequences $\left(x_{n}\right)$ and $\left(y_{n}\right)$ are $K$-equivalent if they $K$-dominate each other.

We will need some information about property $(H)$ and related properties.

Definition 1.2. A Banach space $X$ has property $\left(H_{2}\right)$ if there is a function $C(\cdot, \cdot)$ so that for every $0<\delta<1$ and for every normalized $\lambda$ unconditional basic sequence $\left(x_{i}\right)_{i=1}^{n}$ in $X$ there is a subset $F \subseteq \mathbb{N}$ such that $|F| \geq \delta n$ and $\left(x_{i}\right)_{i \in F}$ is $C(\lambda, \delta)$-equivalent to the unit vector basis of $\ell_{2}^{|F|}$. If $\left(x_{i}\right)_{i \in F}$ is just $C(\lambda, \delta)$-dominated by the unit vector basis of $\ell_{2}^{|F|}$, we say that $X$ has property upper $\left(H_{2}\right)$. Similarly, we define property lower $\left(H_{2}\right)$.

Definition 1.3. A Banach space $X$ is said to have property $(H)$ if there is a function $f(\cdot)$ so that for every normalized $\lambda$-unconditional basic sequence $\left(x_{i}\right)_{i=1}^{n}$ in $X$, we have

$$
\frac{1}{f(\lambda)} n^{1 / 2} \leq\left\|\sum_{i=1}^{n} x_{i}\right\| \leq f(\lambda) n^{1 / 2} .
$$

Similarly, we can define property upper $(H)$ and property lower $(H)$.

The following is clear.

Proposition 1.4. Property upper (resp. lower) $\left(H_{2}\right)$ implies upper (resp. lower) $(H)$. 
We will see later that the converses of Proposition 1.4 fail.

The next result shows that any percentage of the basis will work in the definition of $\left(\mathrm{H}_{2}\right)$. The proof follows from the argument of Pisier [14, Proposition 12.4, p. 193].

Lemma 1.5. For a Banach space $X$, the following are equivalent:

(1) $X$ has property upper (resp. lower) $\left(H_{2}\right)$.

(2) There exists one $0<\delta<1$ satisfying the conclusion of property upper (resp. lower) $\left(H_{2}\right)$.

The corresponding result for property $(H)$ is in [3, Proposition Ae1, p. 14].

LEMma 1.6. For a Banach space $X$, the following are equivalent:

(1) $X$ has property upper (resp. lower) $(H)$.

(2) There is a $0<\delta<1$ so that for every $\lambda$-unconditional basic sequence $\left(x_{i}\right)_{i=1}^{n}$ in $X$ there is a subset $F \subset\{1, \ldots, n\}$ with $|F| \geq \delta$ such that $\left(x_{i}\right)_{i \in F}$ has property upper (resp. lower) $(H)$.

The next theorem is due to Pisier [14, Proposition 12.4].

Proposition 1.7. Every weak Hilbert space has property $\left(H_{2}\right)$.

We also have from Pisier [14, Proposition 10.8, p. 160 and Proposition 11.9, p. 174]:

Proposition 1.8. The following implications hold for a Banach space X:

(1) Weak cotype 2 implies property lower $(H)$.

(2) Weak type 2 implies property upper $(H)$.

The converses of Proposition 1.8 are open questions. However, for Banach lattices it is known that property $(H)$, property $\left(H_{2}\right)$ and being a weak Hilbert space are all equivalent. This is a result of Nielsen and TomczakJaegermann [12].

2. Convexified Tsirelson space. Since there is only a "partial theory" developed for the convexified Tsirelson space $T^{2}$, we will review what we need here.

Notation 2.1. If $E, F$ are sets of natural numbers, we write $E<F$ if for every $n \in E$ and every $m \in F, n<m$. If $E=\{k\}$, we just write $k<F$ for $E<F$.

Definition 2.2. Let $\left(t_{n}\right)_{n=1}^{\infty}$ be the canonical unit vectors in $\mathbb{R}^{\mathbb{N}}$. The convexified Tsirelson space $T^{2}$ (see [3]) is the set of vectors $x=\sum_{n} a_{n} t_{n}$ for 
which the recursively defined norm below is finite:

$$
\|x\|_{T^{2}}=\max \left\{\sup \left|a_{n}\right|, 2^{-1 / 2} \sup \left(\sum_{j=1}^{k}\left\|E_{j} x\right\|_{T^{2}}^{2}\right)^{1 / 2}\right\},
$$

where the second "sup" is taken over all choices

$$
k \leq E_{1}<\ldots<E_{k}
$$

and $E x=\sum_{n \in E} a_{n} t_{n}$.

Remark. It follows from Pisier [14, Chapter 13] ([3, Chapter 1] for the original Tsirelson space) that there exists a norm satisfying (2.1) above.

We will now list the known results for this space (which we will need) and where they can be found. Although many of the results we need have formally been proved for the original Tsirelson space $T$, using the fact that $T^{2}$ is the 2-convexification of $T$ we can easily carry these results over to $T^{2}$. The first result can be found in [3] and [14].

Proposition 2.3. The unit vectors $\left(t_{n}\right)$ form a 1-unconditional basis for $T^{2}$. The space $T^{2}$ is of type 2 and weak cotype 2 but does not contain an infinite-dimensional Hilbert space. Also, the unit vector basis $\left(e_{n}\right)$ of $\ell_{2}$ 1-dominates all subsequences of $\left(t_{n}\right)$. That is, for $x \in T^{2},\|x\|_{T^{2}} \leq\|x\|_{\ell_{2}}$. Finally, if $I \subset\{n, n+1, \ldots\}$ with $|I| \leq n$ and $x=\sum_{n \in I} a_{n} t_{n}$ then $\|x\|_{\ell_{2}} \leq$ $2\|x\|_{T^{2}}$.

We also need [3, Lemma II.1, p. 19]. This lemma is stated in [3] for the original Tsirelson space but the proof works the same in $T^{2}$.

Proposition 2.4. Let $y_{n}=\sum_{i \in I_{n}} a_{i} t_{i}(n=1,2, \ldots)$ be a normalized disjointly supported sequence in $\left(t_{n}\right)$ and let $p_{n}=\min I_{n}$. Then for every sequence $\left(a_{n}\right)$ of scalars we have

$$
\left\|\sum_{n} a_{n} t_{p_{n}}\right\|_{T^{2}} \leq\left\|\sum_{n} a_{n} y_{n}\right\|_{T^{2}} .
$$

Next we need to see which subsequences of the unit vector basis of $T^{2}$ are equivalent to the original basis. To do this we need:

Notation 2.5. The fast growing hierarchy from logic is a family of functions on $\mathbb{N}$ given by: $g_{0}(n)=n+1$, and for $i \geq 0, g_{i+1}(n)=g_{i}^{(n)}(n)$, where for any function $f, f^{(n)}$ is the $n$-fold iteration of $f$. We also set $\exp _{0}(n)=n$ and for $i \geq 1$ and $n \in \mathbb{N}$,

$$
\exp _{i}(n)=2^{\exp _{i-1}(n)} .
$$

Finally we let $\log _{0}(n)=n$, and for any $i \geq 1$ and $n$ large enough so that $\log _{i-1}(n)>0$, let

$$
\log _{i}(n)=\log \left(\log _{i-1}(n)\right) .
$$


The next result is due to Bellenot [1]. He states this result in the original Tsirelson space $T$, but the proof works perfectly well in $T^{2}$.

Proposition 2.6. A subsequence $\left(t_{k_{n}}\right)$ of $\left(t_{n}\right)$ is equivalent to $\left(t_{n}\right)$ if and only if there is a natural number $i$ so that $k_{n} \leq g_{i}(n)$ for all large $n$. Moreover, $\left(t_{k_{n}}\right)$ always 1-dominates $\left(t_{n}\right)$ and there is a constant $K \geq 1$ so that the equivalence constant is $K^{i}$ for the case $k_{n}=g_{i}(n)$.

One important consequence is (see [14] or [3])

Proposition 2.7. For every natural number $i$, every $g_{i}(n)$-dimensional subspace of $\operatorname{span}\left(t_{j}\right)_{j \geq n}$ is $K^{i}$-isomorphic to a Hilbert space and $K^{i}$-complemented in $T^{2}$.

If $X$ is a weak Hilbert space with an unconditional basis, then it follows from [12] that the conclusion of Proposition 2.7 remains true after a suitable permutation of the basis.

The next result comes from [3, Theorem IV.b.3, p. 39]. The theorem there is proved for the regular Tsirelson space but the techniques easily adapt to the convexified space. Also, although the theorem is stated for distances between subspaces, the proof actually checks the equivalence constant of normalized disjointly supported sequences with $n$ elements in $\left(t_{k}\right)$ and the unit vector basis of $\ell_{1}^{n}$. So we state this form here.

Proposition 2.8. Every $n$-dimensional subspace of $T^{2}$ is $K^{i} \log _{i}(n)$ isomorphic to $\ell_{2}^{n}$, for every $i \geq 2$ with $\log _{i}(n) \geq 1$. Moreover, every normalized disjointly supported sequence $\left(x_{j}\right)_{j=1}^{n}$ in $T^{2}$ is $K^{i} \log _{i}(n)$-equivalent to the unit vector basis of $\ell_{2}^{n}$, and if the support of the $x_{j}$ 's lies in $\left(t_{j}\right)_{j=n}^{\infty}$, then $\left(x_{j}\right)_{j=1}^{n}$ is 2-equivalent to the unit vector basis of $\ell_{2}^{n}$.

We need one more result on convexified Tsirelson.

Proposition 2.9. If $x=\sum_{j} a_{j} t_{j} \in T^{2}$, then for all $n \in \mathbb{N}$ and all $i \geq 2$ with $\log _{i}(n) \geq 1$,

$$
\left\|\sum_{j} a_{j} t_{n j}\right\|_{T^{2}} \leq 2 K^{i} \log _{i}(n)\|x\|_{T^{2}} .
$$

Proof. By Propositions 2.6 and 2.8 we have

$$
\begin{aligned}
\left\|\sum_{j} a_{j} t_{n j}\right\|_{T^{2}} & \leq\left\|\sum_{j=1}^{n} a_{j} t_{n j}\right\|_{T^{2}}+\left\|\sum_{j=n+1}^{\infty} a_{j} t_{n j}\right\|_{T^{2}} \\
& \leq\left(\sum_{j=1}^{n}\left|a_{j}\right|^{2}\right)^{1 / 2}+K^{2}\left\|\sum_{j=n+1}^{\infty} a_{j} t_{j}\right\|_{T^{2}}
\end{aligned}
$$




$$
\begin{aligned}
& \leq K^{i} \log _{i}(n)\left\|\sum_{j=1}^{n} a_{j} t_{j}\right\|_{T^{2}}+K^{2}\left\|\sum_{j=n+1}^{\infty} a_{j} t_{j}\right\|_{T^{2}} \\
& \leq 2 K^{i} \log _{i}(n)\|x\|_{T^{2}}
\end{aligned}
$$

where in the second inequality above we use Propositions 2.3 and 2.6 and the fact that $n j \leq g_{2}(j)$ for all $j \geq n+1$; and in the third inequality we have used Proposition 2.8.

3. Symmetric convexified Tsirelson space. There is almost no existing theory for the symmetric convexified Tsirelson space. But there is a theory for the symmetric Tsirelson space. We will list the results we need on this topic. They can be found in [3, Chapter X.E].

Notation 3.1. For $T^{2}$ or $\left(T^{2}\right)^{*}$ we will work with the non-decreasing rearrangement operator $D$. That is, if $x=\sum_{n} a_{n} t_{n}$ then $D x=\sum_{n} a_{n}^{*} t_{n}$ where $\left(a_{n}^{*}\right)$ is the non-decreasing rearrangement of the non-zero $a_{n}$ 's where by non-decreasing we mean the absolute values are non-decreasing.

The construction of [3, Chapters VIII and X.B] shows

Proposition 3.2. Let $\Pi$ denote the group of all permutations of $\mathbb{N}$. There is a constant $K \geq 1$ so that for any $x=\sum_{n} a_{n} t_{n}^{*} \in\left(T^{2}\right)^{*}$ we have

$$
\begin{aligned}
\|x\|_{S\left[\left(T^{2}\right)^{*}\right]} & :=\sup _{\sigma \in \Pi}\left\|\sum_{n} a_{\sigma(n)} t_{n}^{*}\right\|_{\left(T^{2}\right)^{*}} \\
& \leq K\|D x\|_{\left(T^{2}\right)^{*}} \leq K \sup _{\sigma \in \Pi}\left\|\sum_{n} a_{\sigma(n)} t_{n}^{*}\right\|_{S\left[\left(T^{2}\right)^{*}\right]} .
\end{aligned}
$$

We will define the dual space of the symmetric convexified Tsirelson space first, because it is natural in terms of the above.

Definition 3.3. We let $S\left[\left(T^{2}\right)^{*}\right]$ be the family of all vectors for which $\|x\|_{S\left[\left(T^{2}\right)^{*}\right]}$ is finite. Then this is a Banach space with a natural symmetric basis, denoted by $\left(t_{n}^{\mathrm{s} *}\right)$, called the dual space of the symmetric convexified Tsirelson space.

To define the symmetric convexified Tsirelson space we need a result kindly communicated to us by N. J. Kalton.

Let $X$ be a Banach sequence space with the standard unit vector basis $\left(e_{i}\right)$. Define the permutation operators $S_{\sigma}(\xi)=\left(\xi_{\sigma(n)}\right)_{n=1}^{\infty}$ for $\sigma \in \Pi$ and let $L_{k}^{j}$ be the linear map such that $L_{k}^{j}\left(e_{n}\right)=e_{k n+j}$ for all $n \in \mathbb{N}$. Finally we let $c_{00}$ denote the spaces of real sequences which are eventually 0 .

THEOREM 3.4. Suppose $X$ is a Banach sequence space which is $p$-convex and $q$-concave where $1<p<q<\infty$. Suppose $\max _{0 \leq j<k}\left\|L_{k}^{j}\right\| \leq C k^{a}$ where 
$a+p^{-1}<1$. Then

$$
\|\xi\|_{X_{\mathrm{inf}}}=\inf _{\sigma \in \Pi}\left\|S_{\sigma} \xi\right\|_{X}, \quad x \in c_{00}
$$

defines a quasi-norm on $c_{00}$ which is equivalent to a norm. The dual of $X_{\text {inf }}$ is $X_{\mathrm{sup}}^{*}$ where

$$
\|\xi\|_{X_{\text {sup }}^{*}}=\sup _{\sigma \in \Pi}\left\|S_{\sigma} \xi\right\|_{X^{*}}
$$

Proof. Let us start by supposing $x_{1}, \ldots, x_{k} \in c_{00}$ are disjointly supported and that $\sigma_{1}, \ldots, \sigma_{k} \in \Pi$. Then

$$
\begin{aligned}
\left\|x_{1}+\ldots+x_{k}\right\|_{X_{\mathrm{inf}}} & \leq\left\|\sum_{j=1}^{k} L_{k}^{j-1} S_{\sigma_{j}} x_{j}\right\|_{X} \leq\left(\sum_{j=1}^{k}\left\|L_{k}^{j-1} S_{\sigma_{j}} x_{j}\right\|_{X}^{p}\right)^{1 / p} \\
& \leq C k^{a}\left(\sum_{j=1}^{k}\left\|S_{\sigma_{j}} x_{j}\right\|_{X}^{p}\right)^{1 / p} .
\end{aligned}
$$

Now taking an infimum over $\sigma_{j}$ gives

$$
\left\|x_{1}+\ldots+x_{k}\right\|_{X_{\mathrm{inf}}} \leq C k^{a}\left(\sum_{j=1}^{k}\left\|x_{j}\right\|_{X_{\mathrm{inf}}}^{p}\right)^{1 / p} .
$$

Let us use (3.2) first to show that $\|\cdot\|_{X_{\text {inf }}}$ is a quasi-norm. Indeed if $x, y \in c_{00}$ then

$$
\|x+y\|_{X_{\mathrm{inf}}} \leq 2\|\max (|x|,|y|)\|_{X_{\mathrm{inf}}} \leq 2^{a+1} C\left(\|x\|_{X_{\mathrm{inf}}}+\|y\|_{X_{\mathrm{inf}}}\right) .
$$

Next note that (3.2) implies

$$
\left\|x_{1}+\ldots+x_{k}\right\|_{X_{\mathrm{inf}}} \leq C k^{a+1 / p} \max _{1 \leq j \leq k}\left\|x_{j}\right\|_{X_{\mathrm{inf}}} .
$$

From this it follows easily that if $a+1 / p<1 / r<1$ then

$$
\left\|x_{1}+\ldots+x_{k}\right\|_{X_{\text {inf }}} \leq C_{r}\left(\sum_{j=1}^{k}\left\|x_{j}\right\|_{X_{\text {inf }}}^{r}\right)^{1 / r}
$$

for disjoint $x_{1}, \ldots, x_{k}$. Thus we have an upper $r$-estimate for $X_{\text {inf }}$.

It is trivial to show that $X_{\text {inf }}$ has a lower $q$-estimate. Now by $[5$, Theorem 4.1] (a simpler proof is given in [6, Theorem 3.2]), $X_{\text {inf }}$ is lattice-convex and this means that an upper $r$-estimate implies (lattice) $s$-convexity for all $s<r$ (Theorem 2.2 of [5]). Hence $X_{\text {inf }}$ is $r$-convex for every $r$ with $a+1 / p<1 / r$. In particular 1-convexity implies that the quasi-norm is equivalent to a norm. In fact $X_{\text {inf }}^{*}$ is a reflexive Banach space.

Now it is obvious that $X_{\text {inf }} \subset\left(X_{\text {sup }}^{*}\right)^{*}$ and $X_{\text {sup }}^{*} \subset\left(X_{\text {inf }}\right)^{*}$. Hence it follows easily that $\left(X_{\text {inf }}\right)^{*}=X_{\text {sup }}^{*}$. 
REMARK. We can apply the above result to the case of the weighted $\ell_{p}$-space $X$ with $1<p<\infty$, defined by the norm

$$
\|\xi\|_{X}=\left(\sum_{n=1}^{\infty}\left|\xi_{n}\right|^{p} w_{n}\right)^{1 / p}
$$

where $\left(w_{n}\right)$ is an increasing sequence satisfying an estimate of the form

$$
w_{k n} \leq C k^{a} w_{n}
$$

where $a<p-1$. The $X_{\text {inf }}$ is defined by the quasi-norm

$$
\|\xi\|_{X_{\mathrm{inf}}}=\left(\sum_{n=1}^{\infty}\left(\xi_{n}^{*}\right)^{p} w_{n}^{p}\right)^{1 / p}
$$

where $\left(\xi_{n}^{*}\right)$ is the decreasing rearrangement of $\left(\left|\xi_{n}\right|\right)$. In this case $X_{\text {sup }}$ is the Lorentz space $d\left(\left(w_{n}\right)^{-q / p}, q\right)$.

This result can be rephrased. If $\left(v_{n}\right)$ is a positive decreasing sequence satisfying an estimate $v_{n} \leq C k^{b} v_{k n}$ where $b<1$ then $d\left(\left(v_{n}\right), p\right)^{*}$ can be identified with the space of all sequences $\left(\xi_{n}\right)$ so that

$$
\left(\sum_{n=1}^{\infty}\left(\xi_{n}^{*}\right)^{q} v_{n}^{-q / p}\right)^{1 / q}<\infty
$$

This result is a special case of results of Reisner [15].

Proposition VIII.a.8 of [3] states that the decreasing rearrangement operator $D$ is a bounded non-linear operator on the original Tsirelson space $T$. This result then immediately carries over to the 2-convexification of $T$ which is our convexified Tsirelson space $T^{2}$. By Proposition 2.9, Theorem 3.4 holds in this case. We summarize this in the following result:

Proposition 3.5. There is a constant $K \geq 1$ so that for any $x=$ $\sum_{n} a_{n} t_{n} \in T^{2}$ we have

$$
\inf _{\sigma \in \Pi}\left\|\sum_{n} a_{\sigma(n)} t_{n}\right\|_{T^{2}} \leq\|D x\|_{T^{2}} \leq K \inf _{\sigma \in \Pi}\left\|\sum_{n} a_{\sigma(n)} t_{n}\right\|_{T^{2}} .
$$

Moreover, there is a norm $\|\cdot\|_{S\left(T^{2}\right)}$ on the set of vectors for which $\|D x\|<\infty$ satisfying

$$
\frac{1}{K}\|x\|_{S\left(T^{2}\right)} \leq\|D x\|_{T^{2}} \leq K\|x\|_{S\left(T^{2}\right)}
$$

Note that our operator $D$ does not satisfy a triangle inequality, but does with the constant $K$ on the sum side.

Definition 3.6. The symmetric convexified Tsirelson space is the Banach space $S\left(T^{2}\right)$ of vectors for which $\|x\|_{S\left(T^{2}\right)}<\infty$ with natural unit vector basis $\left(t_{n}^{s}\right)$. By Theorem 3.4 this is a reflexive Banach space whose dual space is $S\left[\left(T^{2}\right)^{*}\right]$. 
It is known [3] that every infinite-dimensional subspace of $S\left(T^{2}\right)$ contains a subspace which embeds into $T^{2}$. In particular $S\left(T^{2}\right)$ is a Banach space with a natural symmetric basis which has no subspaces isomorphic to $c_{0}$ or $\ell_{p}$ for $1 \leq p<\infty$. Also $T^{2}$ embeds into $S\left(T^{2}\right)$. Since the unit vector basis of $\ell_{2}$ uniformly dominates all block bases of $\left(t_{n}\right)$ in $T^{2}$, it follows that the unit vector basis of $S\left(T^{2}\right)$ is also dominated by the unit vector basis of $\ell_{2}$.

Proposition 3.7. The space $S\left(T^{2}\right)$ fails property upper $(H)$ (even for disjointly supported elements) and fails property lower $\left(H_{2}\right)$. Hence $S\left(T^{2}\right)$ is not of weak type 2 and not of cotype 2.

Proof. First we check property lower $\left(H_{2}\right)$. Since $\left(t_{n}^{s}\right)$ is symmetric and is dominated by the unit vector basis of $\ell_{2}$, it follows that if this family had subsets dominating the unit vector basis of $\ell_{2}$, then $\left(t_{n}^{s}\right)$ would be equivalent to the unit vector basis of $\ell_{2}$, which is impossible.

For property upper $(H)$, fix $M>1$ and choose a decreasing sequence $\left(a_{i}\right)_{i=1}^{n}$ of non-zero scalars with $\left\|\sum_{i=1}^{n} a_{i} e_{i}\right\|_{\ell_{2}} \geq M$ and $\left\|\sum_{i=1}^{n} a_{i} t_{i}\right\|_{T^{2}}=1$. This can be done by an obvious modification of the construction of [3, Chapter IV]. For all $1 \leq j \leq 2 n$ let

$$
x_{j}=\sum_{i=n j+1}^{n(j+1)} a_{i-n j} t_{i}^{s}
$$

be vectors in $S\left(T^{2}\right)$. So $\left\|x_{j}\right\|_{S\left(T^{2}\right)} \leq K$ for all $1 \leq j \leq 2 n$. Now, $D\left(\sum_{i=1}^{2 n} x_{i}\right)$ $=\sum_{i=1}^{2 n} y_{i}$ where each $y_{i}$ is an appropriate permutation of $x_{i}$. Since the $y_{i}$ 's are disjoint, there is a subset $I \subset\{1, \ldots, 2 n\}$ with $|I| \geq n$ such that each $y_{i}$ with $i \in I$ has its support in $\left(t_{j}^{s}\right)_{j=n}^{\infty}$. Hence,

$$
\begin{aligned}
\left\|\sum_{i=1}^{2 n} x_{i}\right\|_{S\left(T^{2}\right)} & \geq K^{-1}\left\|D\left(\sum_{i=1}^{2 n} x_{i}\right)\right\|_{T^{2}} \geq K^{-1}\left\|\sum_{i \in I} y_{i}\right\|_{T^{2}} \\
& \geq \underset{(1)}{\geq}(2 K)^{-1}\left(\sum_{i \in I}\left\|y_{i}\right\|_{T^{2}}^{2}\right)^{1 / 2} \underset{(2)}{\geq}(4 K)^{-1}\left(\sum_{i \in I}\left\|y_{i}\right\|_{\ell_{2}}^{2}\right)^{1 / 2} \\
& \geq(4 K)^{-1} M|I|^{1 / 2}=(4 K)^{-1} M n^{1 / 2} .
\end{aligned}
$$

In (1) above we use Proposition 2.8, and in (2) we use Proposition 2.3.

Since $M$ was arbitrarily large, it follows that $S\left(T^{2}\right)$ fails upper $(H)$ for disjoint elements.

We shall now need a result essentially due to S. Kwapień. In the form we present it, it is due to W. B. Johnson and it appeared in [7].

Proposition 3.8. There is a function

$$
N(k, \varepsilon)=\left[2 k^{2} / \varepsilon\right]^{k}
$$


such that for any fixed $0<\varepsilon<1$, every order complete Banach lattice $L$, and every $k$-dimensional subspace $F$ of $L$, there are $N=N(k, \varepsilon)$ disjoint elements $\left(x_{j}\right)_{j=1}^{N}$ in $L$ and a linear operator $V: F \rightarrow X=\operatorname{span}\left(x_{j}\right)$ such that for all $x \in F$ we have

$$
\|V x-x\| \leq \varepsilon\|x\| .
$$

Proposition 3.9. There is a constant $K>1$ so that for every subspace $E$ of $S\left(T^{2}\right)$ of dimension $n$, for all $i \in \mathbb{N}$ for which $\log _{i-1}(n)$ exists, we have

$$
d\left(E, \ell_{2}^{n}\right) \leq K^{i} \log _{i-2}(n) .
$$

Moreover, any normalized disjointly supported sequence $\left(x_{i}\right)_{i=1}^{n}$ of vectors in $S\left(T^{2}\right)$ is $K^{i} \log _{i-2}(n)$-equivalent to the unit vector basis of $\ell_{2}^{n}$.

Proof. By giving up one level of logs we may assume by Proposition 3.8 that we are working with a normalized disjointly supported sequence $\left(x_{j}\right)_{j=1}^{n}$ of vectors in $S\left(T^{2}\right)$. We will show that this disjointly supported sequence is $K^{i} \log _{i-2}(n)$-equivalent to the unit vector basis of $\ell_{2}$. Now there is a disjoint set of permutations $y_{j}$ of the $x_{j}$ so that

$$
\begin{aligned}
\left\|\sum_{j=1}^{n} a_{j} x_{j}\right\|_{S\left(T^{2}\right)} & \geq \frac{1}{K}\left\|\sum_{j=1}^{n} a_{j} y_{j}\right\|_{T^{2}} \geq \frac{1}{K}\left\|\sum_{j=1}^{n} a_{j} t_{j}\right\|_{T^{2}} \\
& \geq \frac{1}{K^{i+1} \log _{i}(n)}\left(\sum_{j=1}^{n}\left|a_{j}\right|^{2}\right)^{1 / 2}
\end{aligned}
$$

where in the second inequality above we use Proposition 2.4 and the third uses Proposition 2.8. Also, let $D x_{j}=z_{j}$ and

$$
w_{j}=\sum_{k} z_{j}(k) t_{n(k-1)+j} .
$$

We may asume that $K$ is larger than the type 2 constant of $T^{2}$. By Proposition 2.9 we have

$$
\begin{aligned}
\left\|D \sum_{j=1}^{n} a_{j} x_{j}\right\|_{S\left(T^{2}\right)} & \leq K\left\|\sum_{j=1}^{n} a_{j} w_{j}\right\|_{T^{2}} \leq K^{2}\left(\sum_{j=1}^{n}\left|a_{j}\right|^{2}\left\|w_{j}\right\|_{T^{2}}^{2}\right)^{1 / 2} \\
& \leq K^{2}\left(\sum_{j=1}^{n}\left|a_{j}\right|^{2}\left[2 K^{i} \log _{i}(n)\right]^{2}\right)^{1 / 2} \\
& \leq 2 K^{i+2} \log _{i}(n)\left(\sum_{j=1}^{n}\left|a_{j}\right|^{2}\right)^{1 / 2}
\end{aligned}
$$

where in the second inequality above we used the fact that the type 2 constant of $T^{2}$ is less than or equal to $K$. Now,

$$
d\left(E, \ell_{2}^{n}\right) \leq 2 K^{2 i+3} \log _{i}(n)^{2} \leq K^{i} \log _{i-1}(n) .
$$


The $\log _{i-2}(n)$ in the statement of the theorem comes from the fact that we first applied Proposition 3.8.

Recall that the Maurey-Pisier Theorem (see e.g. [11, p. 85]) states that if $X$ is a Banach space then $\ell_{p_{X}}$ and $\ell_{q_{X}}$ are finitely representable in $X$ where

$$
p_{X}=\sup \{p \mid X \text { is of type } p\}, \quad q_{X}=\inf \{q \mid X \text { is of cotype } q\} .
$$

However, Proposition 3.9 implies that the only $\ell_{p}$-space which is finitely representable in $S\left(T^{2}\right)$ is $\ell_{2}$. This gives the following result.

Corollary 3.10. The space $S\left(T^{2}\right)$ is of type $p$ for all $1 \leq p<2$ and of cotype $q$ for all $q>2$.

Before we go on, we need a criterion for a Banach space to be of weak cotype 2 . We shall say that a Banach space $X$ has property $(P)$ if there is a constant $K$ so that whenever $\left\{x_{1}, \ldots, x_{n}\right\} \subseteq X$ is a finite set with $\max _{1 \leq j \leq n}\left|s_{j}\right| \leq\left\|\sum_{j=1}^{n} s_{j} x_{j}\right\|$ for all $\left(s_{j}\right) \subseteq \mathbb{R}$, then

$$
\sqrt{n} \leq K\left(\int\left\|\sum_{j=1}^{n} g_{j}(t) x_{j}\right\|^{2} d \mu(t)\right)^{1 / 2} .
$$

It was proved by Pisier [14, Proposition 10.8] that if $X$ is of weak cotype 2 , then it has property $(P)$. It turns out that $(P)$ characterizes weak cotype 2 spaces. This fact might be known to specialists but we shall give a short proof here:

Theorem 3.11. If $X$ has property $(P)$, then it is of weak cotype 2 .

Proof. Let $E \subseteq X$ be a finite-dimensional subspace, say $\operatorname{dim}(E)=2 n$. By a result of Bourgain and Szarek [2, Theorem 2] there is a universal constant $C$ and $\left\{x_{1}, \ldots, x_{n}\right\} \subseteq X$ so that for all $\left(s_{j}\right) \subseteq \mathbb{R}$ we have

$$
\max _{1 \leq j \leq n}\left|s_{j}\right| \leq\left\|\sum_{j=1}^{n} s_{j} x_{j}\right\| \leq C\left(\sum_{j=1}^{n}\left|s_{j}\right|^{2}\right)^{1 / 2} .
$$

Using property $(P)$ we get

$$
\sqrt{n} \leq K\left(\int\left\|\sum_{j=1}^{n} g_{j}(t) x_{j}\right\|^{2} d \mu(t)\right)^{1 / 2}
$$

where $K$ is the constant of property $(P)$. Now, (3.7) and the right inequality of (3.6) allow us to use the well-known formulation of Dvoretzky's theorem (see e.g. [11, Theorem 4.2], also [16, pages 25 and 81]) to conclude that there is a universal constant $\eta$ such that if $k \leq \eta K^{-2} C^{-2} n$, then there is a $k$-dimensional subspace $F \subseteq\left[x_{j}\right]$ with $d\left(F, l_{2}^{k}\right) \leq 2$. From [14, Theorem 10.2] it now follows that $X$ is of weak cotype 2 . 
We shall say that a sequence $\left(x_{j}\right)_{j=1}^{n}$ in a Banach space $X$ is 1-separated if $\left\|x_{i}-x_{j}\right\| \geq 1$ for all $1 \leq i, j \leq n, i \neq j$. It follows immediately from Theorem 3.11 that if every 1 -separated sequence in $X$ satisfies (3.5), then $X$ is of weak cotype 2 .

We are now ready to prove that the symmetric convexified Tsirelson space is a weak cotype 2 space with a symmetric basis which is not of cotype 2 . Hence its dual space is a symmetric space which is of weak type 2 but fails to be of type 2 .

Theorem 3.12. The space $S\left(T^{2}\right)$ is a weak cotype 2 space.

Proof. Let $\left(x_{j}\right)_{j=1}^{n}$ be a 1-separated sequence in $S\left(T^{2}\right)$. Without loss of generality we may assume that for all $1 \leq i \leq n$ we have $\left\|x_{i}\right\|_{S\left(T^{2}\right)} \geq 1$. We wish to show that (3.5) holds. If $K$ is a constant which satisfies (1.5) for both $T^{2}$ and $S\left(T^{2}\right)$, and (3.4), then by definition we can find a $\sigma \in \Pi$ so that

$$
\begin{aligned}
\left\|\left(\sum_{j=1}^{n}\left|S_{\sigma} x_{j}\right|^{2}\right)^{1 / 2}\right\|_{T^{2}} & =\left\|S_{\sigma}\left(\sum_{j=1}^{n}\left|x_{j}\right|^{2}\right)\right\|_{T^{2}} \\
& \leq K\left\|\left(\sum_{j=1}^{n}\left|x_{j}\right|^{2}\right)^{1 / 2}\right\|_{S\left(T^{2}\right)}
\end{aligned}
$$

Since $S_{\sigma}$ is an isometry on $S\left(T^{2}\right)$, we can without loss of generality assume that actually $x_{j}=S_{\sigma} x_{j}$ for all $1 \leq j \leq n$.

Put $k=\log \log n$ and let $P_{k}$ be the natural projection of $T^{2}$ onto the span of $\left(t_{j}\right)_{j=1}^{k}$. We now examine two cases.

CASE I: There is a subset $I \subset\{1, \ldots, n\}$ with $|I| \geq n / 2$ so that $\left\|P_{k} x_{j}\right\|_{\ell_{2}}$ $\geq \log k$ for all $j \in I$. Now we compute

$$
\begin{aligned}
\left(\int \| \sum_{j=1}^{n} g_{j}(t)\right. & \left.x_{j} \|_{S\left(T^{2}\right)}^{2} d \mu(t)\right)^{1 / 2} \\
& \geq \frac{1}{(1)}\left\|\left(\sum_{j=1}^{n}\left|x_{j}\right|^{2}\right)^{1 / 2}\right\|_{S\left(T^{2}\right)} \geq \frac{1}{K^{2}}\left\|\left(\sum_{j=1}^{n}\left|x_{j}\right|^{2}\right)^{1 / 2}\right\|_{T^{2}} \\
& \geq \underset{(2)}{\geq} \frac{1}{K^{2}}\left\|\left(\sum_{j=1}^{n}\left|P_{k} x_{j}\right|^{2}\right)^{1 / 2}\right\|_{T^{2}} \\
& \geq \frac{1}{(3)}\left\|\left(\sum_{j \in I}\left|P_{k} x_{j}\right|^{2}\right)^{1 / 2}\right\|_{\ell_{2}} \\
& =\frac{1}{(\log k) K^{3}}\left(\sum_{j \in I}\left\|P_{k} x_{j}\right\|_{\ell_{2}}^{2}\right)^{1 / 2} \underset{(4)}{\geq} \frac{1}{K^{3} \sqrt{2}} \sqrt{n}
\end{aligned}
$$


where inequality (1) follows from equation (1.5); (2) from the fact that $\left\|P_{k}\right\| \leq 1$; (3) from Proposition 2.7; and (4) from our assumption of case I.

CASE II: There is a subset $I \subset\{1, \ldots, n\}$ with $|I| \geq n / 2$ so that $\left\|P_{k} x_{j}\right\|_{\ell_{2}} \leq \log k$ for all $j \in I$. In this case we make the following claim:

Claim. There is a subset $J \subset I$ with $|J| \geq n / 4$ so that for all $j \in J$,

$$
\left\|\left(I-P_{k}\right) x_{j}\right\|_{T^{2}} \geq \frac{1}{8 K} .
$$

If not, there is a set $J$ as above with

$$
\left\|\left(I-P_{k}\right) x_{j}\right\|_{T^{2}} \leq \frac{1}{8 K} .
$$

By a standard volume comparison argument (see e.g. [11, Lemma 2.6] or [14, Lemma 4.16]) the cardinality of a set of points which are $1 /(4 K)$ apart in a ball of radius $\log k$ in $k$-dimensional Hilbert space is at most $(1+8 K \log k)^{k}$, which by our choice of $k$ is less than or equal to $n / 4$ (at least for large $n$ ). Hence there exist $i, j \in J, i \neq j$, so that

$$
\left\|P_{k}\left(x_{i}-x_{j}\right)\right\|_{\ell_{2}} \leq \frac{1}{4 K} .
$$

Now we compute using our assumptions and Proposition 2.3:

$$
\begin{aligned}
\left\|x_{i}-x_{j}\right\|_{S\left(T^{2}\right)} & \leq K\left\|x_{i}-x_{j}\right\|_{T^{2}} \\
& \leq K\left\|P_{k}\left(x_{i}-x_{j}\right)\right\|_{T^{2}}+K\left\|\left(I-P_{k}\right) x_{i}\right\|_{T^{2}}+K\left\|\left(I-P_{k}\right) x_{j}\right\|_{T^{2}} \\
& \leq K\left\|P_{k}\left(x_{i}-x_{j}\right)\right\|_{\ell_{2}}+K \frac{1}{8 K}+K \frac{1}{8 K} \leq K \frac{1}{4 K}+\frac{1}{4}=\frac{1}{2} .
\end{aligned}
$$

This contradicts our 1-separation assumption. So the claim holds.

Now by the claim, the beginning of the proof, (1.5) and Proposition 2.7 we get

$$
\begin{aligned}
\left(\int\left\|\sum_{j=1}^{n} g_{j}(t) x_{j}\right\|_{S\left(T^{2}\right)}^{2}\right. & d \mu(t))^{1 / 2} \\
& \underset{(1)}{\geq} \frac{1}{K^{2}}\left\|\left(\sum_{j=1}^{n}\left|x_{j}\right|^{2}\right)^{1 / 2}\right\|_{T^{2}} \\
& \underset{(2)}{\geq} \frac{1}{K^{2}}\left\|\left(I-P_{k}\right)\left(\sum_{j \in J}\left|x_{j}\right|^{2}\right)^{1 / 2}\right\|_{T^{2}} \\
& \underset{(3)}{\geq} \frac{1}{K^{3}}\left(\int\left\|\sum_{j=1}^{n} g_{j}(t)\left(I-P_{k}\right) x_{j}\right\|_{T^{2}}^{2} d \mu(t)\right)^{1 / 2}
\end{aligned}
$$




$$
\begin{aligned}
& \underset{(4)}{\geq} \frac{1}{K^{5}}\left(\sum_{j \in J}\left\|\left(I-P_{k}\right) x_{j}\right\|_{T^{2}}^{2}\right)^{1 / 2} \\
& \geq \frac{1}{(5)}\left(\sum_{j \in J}\left(\frac{1}{8 K}\right)^{2}\right)^{1 / 2} \geq \frac{|J|^{1 / 2}}{8 K^{6}} \geq \frac{\sqrt{n}}{16 K^{6}},
\end{aligned}
$$

where (1) follows from $(1.5) ;(2)$ follows from the fact that $\left\|I-P_{k}\right\|=1 ;(3)$ holds because $T^{2}$ is type 2 with constant (we assume) less than or equal to $K$; (4) follows from the fact that $k=\log \log n$ and $\left(\left(I-P_{k}\right) x_{n}\right)$ is supported on $\left(t_{i}\right)_{i=k}^{\infty}$ and Proposition 2.7; and (5) is our Claim. This completes the proof.

As a corollary we obtain:

COROLlary 3.13. Even for Banach lattices, property upper $(H)$ and the weak type 2 property do not imply the upper $\left(H_{2}\right)$ property. Similarly, property lower $(H)$ and the weak cotype 2 property do not imply the lower $\left(\mathrm{H}_{2}\right)$ property.

\section{References}

[1] S. Bellenot, The Banach space $T$ and the fast growing hierarchy from logic, Israel J. Math. 47 (1984), 305-313.

[2] J. Bourgain and S. J. Szarek, The Banach-Mazur distance to the cube and the Dvoretzky-Rogers factorization, Israel J. Math. 62 (1988), 169-180.

[3] P. G. Casazza and T. J. Shura, Tsirelson's Space, Lecture Notes in Math. 1363, Springer, Berlin, 1989.

[4] W. B. Johnson, A reflexive Banach space which is not sufficiently Euclidean, Studia Math. 60 (1976), 187-204.

[5] N. J. Kalton, Convexity conditions for nonlocally convex lattices, Glasgow Math. J. 25 (1984), 141-152.

[6] N. J. Kalton and S. J. Montgomery-Smith, Set-functions and factorization, Arch. Math. (Basel) 61 (1993), 183-200.

[7] J. Lindenstrauss and L. Tzafriri, The uniform approximation property in Orlicz spaces, Israel J. Math. 23 (1976), 142-155.

[8] - - - Classical Banach Spaces I. Sequence Spaces, Ergeb. Math. Grenzgeb. 92, Springer, Berlin, 1977.

[9] —, - Classical Banach Spaces II. Function Spaces, Ergeb. Math. Grenzgeb. 97, Springer, Berlin, 1979.

[10] V. D. Milman and G. Pisier, Banach spaces with a weak cotype 2 property, Israel J. Math. 54 (1986), 139-158.

[11] V. D. Milman and G. Schechtman, Asymptotic Theory of Finite Dimensional Normed Spaces, Lecture Notes in Math. 1200, Springer, New York, 1986.

[12] N. J. Nielsen and N. Tomczak-Jaegermann, Banach lattices with property $(H)$ and weak Hilbert spaces, Illinois J. Math. 36 (1992), 345-371.

[13] G. Pisier, Weak Hilbert spaces, Proc. London Math. Soc. 56 (1988), 547-579. 
[14] G. Pisier, The Volume of Convex Bodies and Banach Space Geometry, Cambridge Tracts in Math. 94, Cambridge Univ. Press, Cambridge, 1989.

[15] S. Reisner, On the duals of Lorentz function and sequence spaces, Indiana Univ. Math. J. 31 (1982), 65-72.

[16] N. Tomczak-Jaegermann, Banach-Mazur Distances and Finite-Dimensional Operator Ideals, Pitman Monographs Surveys Pure Appl. Math. 38, Longman Sci. Tech., 1989.

Department of Mathematics

University of Missouri

Columbia, MO 65211, U.S.A.

E-mail: pete@casazza.math.missouri.edu

Department of Mathematics and Computer Science University of Southern Denmark Campusvej 55

DK-5230 Odense M, Denmark

E-mail: njn@imada.sdu.dk

Received May 2, 2001

Revised version September 20, 2002 